\title{
Conservación y actividad reproductiva de tortuga lora (Lepidochelys olivacea) en la playa de anidación solitaria Punta Banco, Pacifico Sur de Costa Rica. Recomendaciones de manejo a través de dieciséis años de monitoreo
}

\author{
Sandra Viejobueno Muñoz ${ }^{1 *} \&$ Randall Arauz ${ }^{1}$ \\ 1. Programa Restauración de Tortugas Marinas (PRETOMA), San Jose, Costa Rica; sandra.vm7@gmail.com, \\ rarauz@pretoma.org \\ * Correspondencia
}

Recibido 11-VIII-2014. Corregido 20-XI-2014. Aceptado 22-XII-2014.

\begin{abstract}
Conservation and reproductive activity of Olive Ridley sea turtles (Lepidochelys olivacea) in Punta Banco, a solitary nesting beach in South Pacific Costa Rica: Management recommendations after sixteen years of monitoring. Sea turtle nesting activity was monitored in Punta Banco, South Pacific Costa Rica uninterruptedly for sixteen years, accompanied by conservation activities such as nest relocation in hatcheries. Hatching success evaluations were held for "in situ" and relocated nests. We hereby evaluate the reproductive biology of the olive ridley sea turtle (Lepidochelys olivacea), by far the most common turtle sea turtle nesting in Punta Banco ( $98 \%$ of observed turtles). Daily monitoring of nesting activities was held every year from July to December, from 1996 to 2011. We tagged a total of 1239 turtles and recorded 4130 nesting events. The number of recorded nesting events per nesting season ranged from 239 in 1996 to 402 in 1999, whereas the number of observed females ranged from 18 in 1996 to 146 in 1999. Nesting females had an average curved carapace length of $66.8 \mathrm{~cm}$ and an average curve carapace width of $70.7 \mathrm{~cm}$. The average clutch size was 96.7 , with an observed frequency of 1.07 nests/female and an nesting interval of 19.95 days. Hatching success of "in situ" nests was $61.38 \%$, whereas hatching success for nests relocated into hatcheries was $77.9 \%$. The L. olivacea population in Punta Banco beach displays a a positive trend. Rev. Biol. Trop. 63 (Suppl. 1): 383-394. Epub 2015 April 01.
\end{abstract}

Key words: Marine turtle, nesting beach, Lepidochelys olivacea, pacific coast of Costa Rica.

Recientemente se ha observado un creciente interés en la realización de evaluaciones objetivas del estatus de las poblaciones de tortugas marinas, siendo sumamente importante para ello la publicación de los resultados obtenidos en los diferentes programas de monitoreo locales y regionales en playas de anidación (Broderick, Franenstein, Glen \& Hays, 2006). Para el caso de la costa Pacífica de Costa Rica, históricamente se han registrado las anidaciones de cuatro de las ocho especies de tortugas marinas reconocidas en el mundo: tortuga lora (Lepidochelys olivacea), tortuga verde (Chelonia mydas), tortuga carey (Eretmochelys imbricata) y tortuga baula (Dermochelys coriacea).
Todas estas especies están actualmente listadas como amenazadas de extinción por la Unión Internacional para la Conservación de la Naturaleza (UICN) debido a la reducción drástica de sus poblaciones en el último siglo, a consecuencia principalmente de múltiples factores antrópicos: pesca de hembras anidadoras en playas de anidación y rutas migratorias, saqueo de nidos, así como la captura incidental en redes de pesca industrial y artesanal de juveniles, subadultos y adultos (UICN, 2013).

La especie L. olivacea, considerada como la tortuga marina más pequeña y abundante en el mundo (Limpus, 1995), es el quelonio de mayor frecuencia de observación en la 
costa Pacífica costarricense, la cual tiene una extensión de $1016 \mathrm{~km}$. Las hembras adultas pueden realizar anidaciones solitarias o en arribadas (anidación masiva o sincronizada de cientos o miles de tortugas durante varios días consecutivos), siendo playa Ostional y Nancite los dos sitos más importantes de arribadas en el país (Cornelius, Alvarado, Castro, Mata \& Robinson, 1992; Spotila, 2004; Plotkin, 2007). Diversos proyectos de conservación han sido desarrollados para evaluar y monitorear anualmente la actividad de anidación de hembras adultas y el éxito de eclosión de nidadas para poblaciones de tortuga lora a lo largo de la costa Pacífica del país. Sin embargo, los retos impuestos en el seguimiento de poblaciones de tortugas marinas varían directamente con el tamaño de la población, pues se requieren de niveles bajos de variabilidad no explicada y muestreos que sean lo más completos posibles, a fin de incrementar la robustez estadística de las estimaciones anuales para las poblaciones de estos quelonios (Bjorndal, Wetherall, Bolten \& Mortimer, 1999; Russo \& Girondot, 2009; Vera \& Buitrago, 2012), por lo que el valor real de los censos en playas de anidación se sitúa en el seguimiento a la condición de poblaciones específicas a través del establecimiento a largo plazo de un registro estandarizado, repetible y estadísticamente riguroso de los eventos de anidación (Schroeder \& Murphy, 1999).

El proyecto de conservación y monitoreo de tortugas marinas en playa Punta Banco, coordinado por el Programa Restauración de Tortugas Marinas (PRETOMA), con más de una década de datos de marcaje e información biológica (Viejobueno, Adams \& Arauz, 2013), representa una sólida fuente de conocimiento acerca de la ecología de L. olivacea en playas de anidación solitarias en Costa Rica y el Pacífico. La ONG PRETOMA, también ha realizado actividades sociales y educativas en el pueblo de Punta Banco y alrededores, trabajando directamente con los grupos sociales que interactúan con las tortugas marinas, tales como comunidades costeras y pescadores, respetando su cultura y necesidades económicas. Se han impartido clases de educación ambiental en las escuelas, campañas de concientización y talleres para contribuir, promover y organizar programas que permitan tomar medidas para la protección, restauración y conservación de las especies de tortuga y otra fauna marina.

En el presente estudio, presentamos los métodos de campo utilizados por PRETOMA y el análisis de datos en la actividad de anidación de L. olivacea en playa Punta Banco, durante las temporadas reproductivas de 1996 a 2011.

La investigación representa el primer estudio realizado a largo plazo de la ecología de anidación y esfuerzo reproductivo de tortugas marinas en la playa solitaria Punta Banco, Pacífico Sur de costa Rica.

\section{MATERIALES Y MÉTODOS}

Área de estudio: Punta Banco es una playa de arena negra utilizada para la anidación por tortugas marinas, y se encuentra localizada al sur de la costa Pacífica de Costa Rica, en la Península de Osa a $65 \mathrm{~km}$ al sur de Golfito ( $8^{\circ} 21^{\prime} 13.76^{\prime \prime} \mathrm{N}-8^{\circ} 08^{\prime} 06.44^{\prime \prime}$ W; Fig. 1), y contigua a un pueblo pequeño y remoto del mismo nombre, con aproximadamente 200 habitantes.

Toma de datos: Desde 1996 y hasta el año 2011, durante los meses de julio a diciembre de cada temporada reproductiva, se realizaron actividades de patrullaje en la playa (dos por noche de tres horas de duración cada una) siguiendo los métodos estándar propuestos por Eckert, Bjorndal, Abreu-Grobois y Donnelly (2000) para la documentación de los eventos de anidación de tortugas marinas. Las labores de patrullaje fueron realizadas por vecinos de Punta Banco, debidamente capacitados, acompañados por pasantes universitarios encargados del manejo técnico de los datos y voluntarios nacionales e internacionales.

Todos los encuentros con hembras anidadoras fueron registrados como "eventos de anidación", los cuales podían corresponder a un evento de anidación exitosa (si se confirmaba la oviposición o puesta de huevos por la hembra anidadora) o no exitosa (considerada 


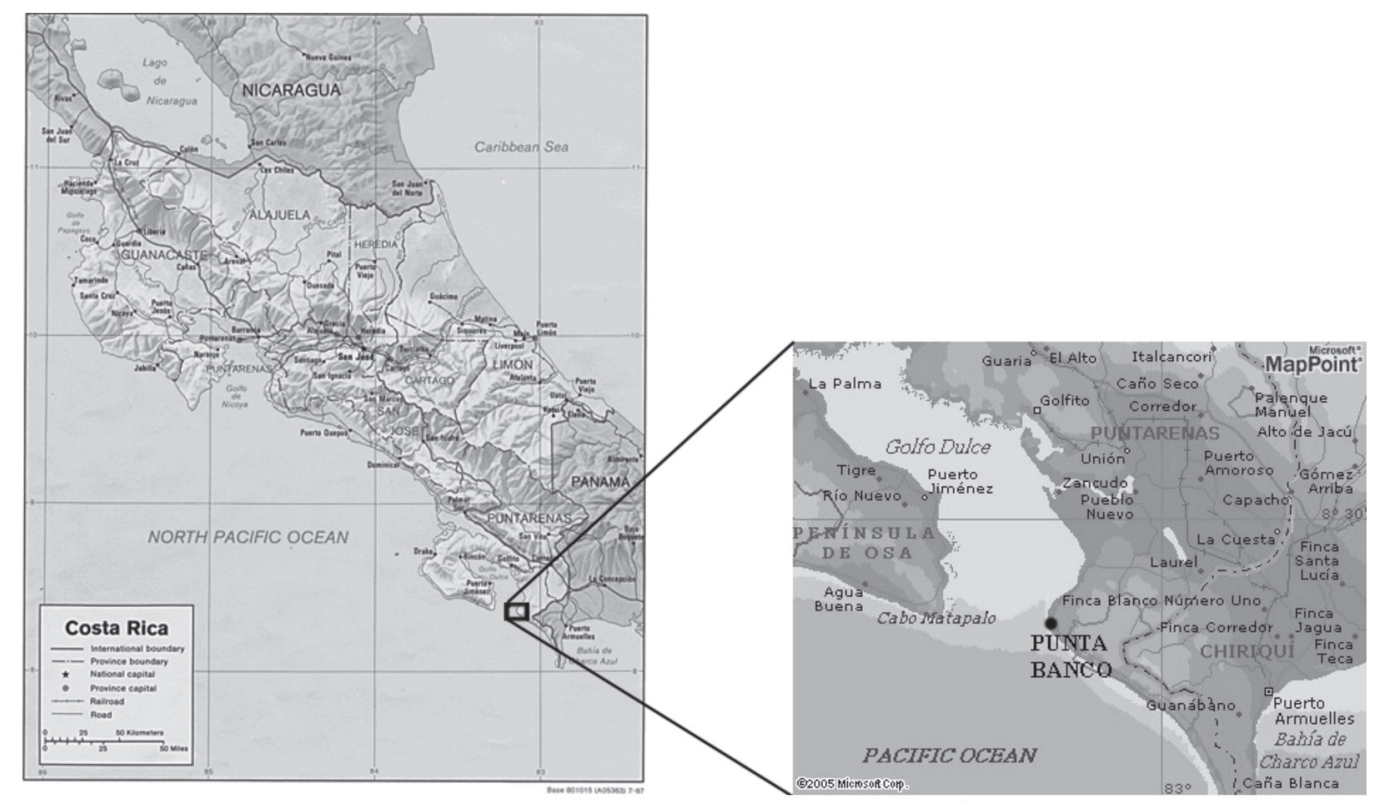

Fig. 1. Ubicación de Playa Punta Banco en el Pacífico Sur de Costa Rica.

Fig. 1. Localization of Punta Banco Beach Southern Pacific Costa Rica.

como una salida falsa o nido abortado, donde la hembra a pesar de construir un nido no realizó la puesta de huevos) (Miller, 2000). Para todos los casos fueron anotados la fecha y hora del evento observado, así como la identificación de la especie correspondiente. Todas las hembras observadas fueron revisadas en busca de marcas previas o cicatriz de marcado anterior, lo cual les identificaba como hembras remigrantes. Las hembras sin placas previas fueron marcadas en las aletas delanteras con marcas metálicas INCONEL $\mathrm{N}^{\circ} 681$ (National Band \& Tag Company, Newport, KY, USA) durante el proceso de oviposición o cobertura de la nidada (Piedra, Vélez, Dutton, Possardt \& Padilla, 2007). Cada marca cuenta con un código único alfanumérico, así como la inscripción "Pretoma, Costa Rica, (506) 22415227” en su reverso para facilitar la identificación y procedencia de los ejemplares con otros proyectos de monitoreo y conservación. Los datos morfométricos largo curvo del caparazón (LCC) y ancho curvo del caprazón (ACC) fueron tomados usando una cinta métrica flexible de $150 \mathrm{~cm}$ de longitud (Bolten, 1999).

El marcado de las hembras anidadoras permitió la estimación de los parámetros reproductivos: Frecuencia de Puesta Observada (FPO), Intervalo de Puesta Observado (IPO) e Intervalo de Remigración (IR). La FPO fue definida como el número promedio de oviposiciones observadas durante la misma temporada por individuo (Alvarado \& Morphy, 1999; Ferrer, Díaz \& Díaz, 2007). El IPO fue definido como el número promedio de días entre una puesta exitosa y el primer intento subsecuente de anidación para una hembra adulta (Ferrer et al., 2007). Estos cálculos excluyeron intervalos menores de seis días, así como los intentos de anidación abortados, de acuerdo a Miller (1997), quien determinó para tortugas marinas un mínimo periodo de interanidación de seis días. El Intervalo de Remigración (IR) (número de años entre dos subsecuentes temporadas de anidación para una hembra) fue determinado usando como referencia la documentación 
del último evento de anidación para cada hembra que aparece registrada en la base de datos de PRETOMA.

Manejo de nidadas: Debido a la problemática del alto saqueo de huevos de tortuga en la comunidad de Punta Banco, se utilizó un vivero como herramienta para proteger las nidadas. Los primeros años de estudio se procedió a dejar algunos nidos in situ, la mayor parte de estos nidos fueron saqueados antes de llegar a su eclosión, y los restantes no pudieron ser monitorizados. Por este motivo se procedió a trabajar con viveros para mayor seguridad y protección de las nidadas. Los datos reportados en este informe corresponden a los nidos relocalizados en el vivero. Los huevos fueron colectados en bolsas plásticas y transportados a un vivero localizado en el centro de la playa, $\left(8^{\circ} 21^{\prime} 14^{\prime \prime} \mathrm{N}-83^{\circ} 08^{\prime} 02.80^{\prime \prime} \mathrm{W}\right)$ justo por detrás de la línea de marea, siempre dentro de las primeras seis horas posteriores al proceso de oviposición (Miller, 2000). La construcción del vivero se realizó en un área cerrada, detrás de la línea de marea, rodeada por una malla de $2 \mathrm{~m}$ de altura y enterrada a $40 \mathrm{~cm}$ de profundidad, para evitar que los animales pudiesen entrar. A modo de manejo, la arena del vivero fue cambiada y filtrada cada año para evitar la proliferación de bacterias y hongos en las nidadas durante el proceso de incubación. (CIT, 2008).

La determinación del tamaño de las nidadas fue realizada mediante el conteo manual de los huevos y considerando para ello solo a los huevos normales (Rees, Saad \& Jony, 2005). El período de incubación fue calculado como el número de días entre la oviposición y la emergencia de la primera cría del nido (Godfrey \& Mrosovsky, 1997). Los nidos en vivero fueron excavados y examinados de tres a cinco días después de la emergencia de las crías, y su contenido se clasificó según Sarti-Martínez (2002) en: número de crías vivas y muertas dentro del nido, número de crías eclosionando vivas o muertas, número de cascarones vacíos, huevos sin eclosionar con y sin embriones, huevos depredados y huevos sin desarrollo aparente (HSDA). Para todas las nidadas fueron calculados los éxitos de eclosión (porcentaje de crías que eclosionan o rompen la cascara respecto al número total de huevos depositados) y emergencia (número de crías vivas y muertas que eclosionaron y alcanzaron la superficie de la playa (Miller, 2000). El éxito de reclutamiento de nidadas para cada temporada fue definido como el promedio del número total de crías liberadas/número de huevos (Sarti-Martínez et al., 2007). El conteo del número total de huevos no eclosionados era realizado por los voluntarios que había en el proyecto, los cuales cambiaron cada mes. Debido a estos cambios de personal durante cada temporada, los datos recolectados de los huevos no eclosionados y HSDA recolectados en cada temporada no se han considerado lo suficientemente fiables y no han sido añadidos en este estudio.

Análisis estadístico: Se determinaron las medidas de tendencia central y dispersión para cada uno de los parámetros reproductivos estudiados por temporada. Para evaluar la normalidad de los datos fue utilizado el estadístico Shapiro-Wilk. Para evaluar las diferencias significativas para las dimensiones corporales entre años se usó una prueba t-test, ya que no estamos interesados en la dirección de la diferencia sino solo en saber si difieren entre años. Análisis de correlación Spearman fueron realizados para evaluar la relación entre las dimensiones de las hembras anidadoras (LCC) con la frecuencia de puesta y el tamaño de la nidada, así como las relaciones entre el tamaño y el período de incubación de las nidadas. Todos los análisis fueron realizados con un nivel de significancia de 0,05 en el programa estadístico SPSS versión 16 y Microsoft Office Excel 2007.

\section{RESULTADOS}

Programa de marcaje y morfometría de Lepidochelys olivacea: Durante los 16 años de estudio fueron muestreadas un total de 2401 noches en playa Punta Banco, en las cuales 
se observaron y se marcaron 1265 tortugas marinas, de las cuales 1239 correspondieron a la especie L. olivácea. Fueron observadas siete tortugas L.olivacea con marcaje previo correspondiente a otras playas de anidación: tres de ellas habían sido marcadas en otro proyecto de PRETOMA en Playa Caletas, Guanacaste, una de ellas había sido marcada en el Refugio de Vida Silvestre Camaronal, Guanacaste y de las otras tres tortugas no fueron identificadas sus placas de procedencia (placas no identificadas fueron: WP458, WP560 y XT738). El tamaño promedio observado (Cuadro 1) para individuos de $L$. olivacea fue LCC $66.8 \pm 0.9$ (LCC mín=65.6cm; LCC máx=69.13cm) y ACC de 70.79 \pm 0.6 (ACC mín=69.8cm; ACC máx $=71,86 \mathrm{~cm})$ sin observación de diferencias significativas entre temporadas para las dimensiones de los animales (T-test; LCC: $\mathrm{p}=1.961806, \quad \mathrm{P}=>0.05 ; \quad$ ACC: $\mathrm{p}=1.961806$, $\mathrm{P}=>0.05)$.

Características reproductivas de $\boldsymbol{L}$. olivacea: Fueron observados un total de 4130 eventos de anidación, 2535 (61.38\%) nidadas éxitosas. De los 1595 eventos de anidación no exitosa el $69.10 \%$ correspondió a salidas falsas y un $30.90 \%$ a nidos abortados durante el proceso de construcción u ovoposición. Las horas con mayor número de eventos están comprendidas entre las 21 y las $00 \mathrm{~h}$ y se registró un mayor porcentaje de salidas de animales entre las 21 y las $22 \mathrm{~h}(14 \%)$. Los eventos de anidación por año variaron de 115 a 402 eventos/año (72-231 nidadas/año; Cuadro 1). La Fig. 2 muestra el número de eventos observados por quincena para los 16 años analizados, siendo septiembre el mes de mayor actividad reproductiva, con un

CUADRO 1

Número de hembras anidadoras y eventos de anidación de tortuga lora (Lepidochelys olivacea) observados por temporada en Punta Banco

TABLE 1

Number of nesting females and nesting events of Ridley sea turtle (Lepidochelys olivacea) observed per season in Punta Banco

\begin{tabular}{cccccccccc} 
Temporada (\#NM) & \# Hembras & N $^{\circ}$ H.R & I.R & LCC & ACC & \# E.A & \# N.E & FPO & IPO \\
$1996(101)$ & 18 & - & - & 67 & 71 & 239 & 150 & 1 & - \\
$1997(127)$ & 80 & 2 & 1 & 67.43 & 70.84 & 177 & 133 & 1.14 & 19 \\
$1998(177)$ & 41 & 0 & - & & & 115 & 72 & 1.1 & 16.7 \\
$1999(153)$ & 146 & 3 & 1,7 & & & 402 & 231 & 1.15 & 31.3 \\
$2000(157)$ & 126 & 6 & 1,4 & & & 286 & 202 & 1.1 & 19.5 \\
$2001(157)$ & 92 & 7 & 1,6 & 66.7 & 71.8 & 239 & 140 & 1.06 & 17.3 \\
$2002(153)$ & 73 & 5 & 2 & 66.87 & 71.47 & 209 & 141 & 1.08 & 16.4 \\
$2003(153)$ & 76 & 4 & 2,5 & 67.92 & 70.96 & 189 & 139 & 1.05 & 23.2 \\
$2004(153)$ & 56 & 2 & 3 & 67.29 & 70.9 & 200 & 103 & 1.12 & 17.6 \\
$2005(155)$ & 67 & 7 & 2,3 & 67.12 & 70.72 & 231 & 138 & 1.15 & 18.9 \\
$2006(155)$ & 97 & 5 & 2,6 & 65.93 & 70.34 & 381 & 210 & 1.1 & 19.6 \\
$2007(150)$ & 119 & 8 & 2,6 & 66 & 69.8 & 365 & 204 & 1.08 & 21 \\
$2008(151)$ & 97 & 7 & 1,2 & 69.13 & 71.86 & 335 & 213 & 0.95 & 22 \\
$2009(153)$ & 106 & 1 & 2,0 & 66.25 & 70.68 & 269 & 167 & 1.09 & 23.1 \\
$2010(153)$ & 62 & 6 & 1,3 & 66.22 & 69.96 & 245 & 163 & 0.98 & 21 \\
$2011(153)$ & 48 & 2 & & 65.6 & 70.04 & 248 & 129 & 1.06 & 12.3 \\
Promedio & 81.5 & 4.5 & 1.93 & 66.8 & 70.79 & 258.1 & 158.4 & 1.07 & 19.95 \\
\hline
\end{tabular}

\#NM=número de noches muestreadas; $\mathrm{N}^{\circ} \mathrm{HR}=$ Número de hembras remigrantes; $\mathrm{IR}=$ Intervalo de remigración (años); LCC=Largo curvo del caparazón $(\mathrm{cm})$; ACC=Ancho curvo del caparazón $(\mathrm{cm})$; \#EA=número de eventos de anidación; \#NE=Número de nidadas exitosas; FPO=Frecuencias de puesta observada; IPO=Intervalo de puesta observado (días). 


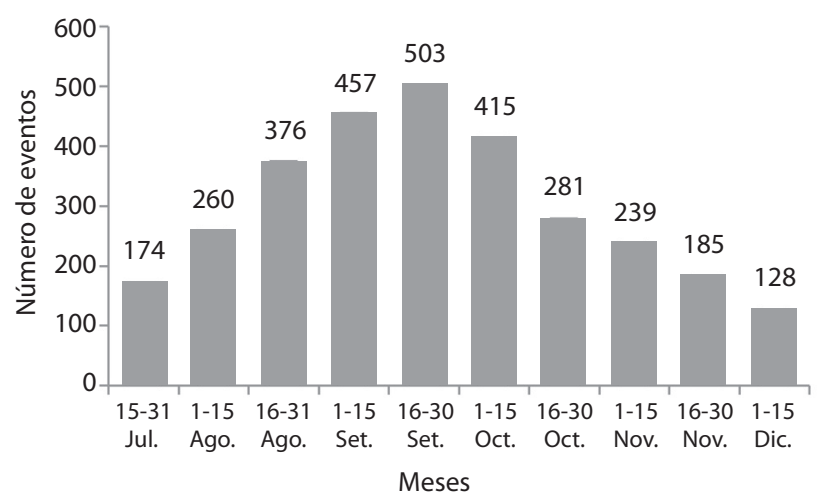

Fig. 2. Suma de todos los años desde 1996 hasta 2011 de la actividad de anidación por quincenas para L.olivacea, Punta Banco, Costa Rica.

Fig. 2. Summary of total years from 1996 to 2011 of nesting activity recorded during 15 day intervals for L.olivacea, Punta Banco, Costa Rica.

total de 503 (16.7\%) eventos de anidación. Para playa Punta Banco entre 1996-2011, con base a los eventos observados para 240 hembras reanidadoras en la misma temporada, la FPO fue de $1.07 \pm 0.06$ veces y el IPO de $19.95 \pm 4.04$ días (Cuadro 1). Se encontró una relación linear negativa entre las dimensiones de las hembras anidadoras (LCC y ACC) y las FPO por temporadas de L. olivacea (LCC: $\mathrm{r}=-0.29$; ACC $\mathrm{r}=-0.19, \mathrm{p}<0.05, \mathrm{n}=13)$.

Manejo de nidadas de L. olivacea: El tamaño promedio por temporada para las nidadas fue de 96.76 \pm 5.3 huevos (Tamaño mín=89.4; Tamaño máx=1 055 n=14; Cuadro 2). Se encontró una relación linear positiva entre las dimensiones de las hembras anidadoras (LCC y ACC) y el tamaño de las nidadas por temporadas (LCC: $\mathrm{r}=0.24$ ACC $\mathrm{r}=0.13$, $\mathrm{p}<0.05, \mathrm{n}=14)$.

Del total de 2535 nidos registrados en playa Punta Banco durante 16 años de estudio, $832(32.88 \%)$ permanecieron in situ y 1703 $(67.12 \%)$ fueron relocalizados en el vivero construido. Para las nidadas in situ se reportaron un total de ocho depredaciones naturales y 566 saqueos de huevos (Cuadro 2). El período de incubación fue de $59 \pm 6.3$ días (período mín=49, período máx $=68.657 \mathrm{n}=16$ ) para nidadas en vivero. Se encontró una relación linear negativa entre el tamaño de las nidadas y los periodos de incubación por temporadas $(\mathrm{r}=-0.6, \mathrm{p}<0.05, \mathrm{n}=16)$. Los valores promedios para los éxitos de eclosión, emergencia y reclutamiento por temporada para nidadas en vivero son reportados en el Cuadro 2.

Las 1703 nidadas (154001 huevos) en vivero fueron revisadas post emergencia de crías, y su contenido clasificado como $77.93 \%$ (120015) huevos eclosionados y 22.06\% (33 986) huevos no eclosionados. Del total de huevos que llegaron a eclosionar: $76.54 \%$ (117 886) correspondieron a crías eclosionando vivas (encontradas vivas fuera y dentro del nido) y $1.38 \%$ (2 129) correspondieron a crías eclosionando muertas. Un total de 117886 (76.54\%) crías de L. olivacea lograron emerger del nido y fueron liberadas al mar en buenas condiciones físicas.

\section{DISCUSIÓN}

El proyecto de monitoreo de tortugas marinas de playa Punta Banco es uno de los más antiguos de Costa Rica, entre los que se incluyen los proyectos del Parque Nacional Tortuguero (creado en 1975) y el Parque Nacional Marino las Baulas (creado en 1991), siendo el proyecto de anidación solitaria de 
CUADRO 2

Manejo de nidadas de tortuga lora (Lepidochelys olivacea) por temporada, desde 1996 a 2011, en Punta Banco

TABLE 2

Clutches management of Ridley sea turtle (Lepidochelys olivacea) per season, since 1996 to 2011, in Punta Banco

\begin{tabular}{ccccccccc}
$\begin{array}{c}\text { Temporada } \\
\text { (\# NM) }\end{array}$ & \# N.D. & \#N.S. & T. N. \pm DE & \multicolumn{7}{c}{ Nidadas en vivero } \\
$1996(101)$ & - & 24 & $102.5 \pm 21.9$ & 126 & 56 & 72.9 & 73 & 72.9 \\
$1997(127)$ & - & 33 & - & 100 & 49 & 47.1 & 47.1 & 47.1 \\
$1998(177)$ & - & 22 & $103 \pm 22.2$ & 50 & 51 & 82.6 & 80.5 & 80.5 \\
$1999(153)$ & - & 75 & $101 \pm 21.2$ & 103 & 57 & 77.5 & 76 & 76 \\
$2000(157)$ & - & 22 & $105 \pm 18.3$ & 140 & 55 & 83.7 & 83.2 & 83.1 \\
$2001(157)$ & - & 35 & $95.8 \pm 24.1$ & 91 & 52.69 & 81.5 & 80.8 & 80.8 \\
$2002(153)$ & - & 24 & - & 110 & 54 & 80.6 & 77.5 & 77.5 \\
$2003(153)$ & - & 33 & $100.4 \pm 22.6$ & 82 & 67.72 & 51.4 & 49.5 & 49.5 \\
$2004(153)$ & - & 10 & $99.22 \pm 21.3$ & 91 & 60.87 & 89.6 & 71.6 & 87 \\
$2005(155)$ & - & 17 & $96.8 \pm 20.6$ & 109 & 65.9 & 84 & 77 & 82.3 \\
$2006(155)$ & 4 & 35 & $95.58 \pm 22.2$ & 141 & 60 & 87 & 84.3 & 86.9 \\
$2007(150)$ & - & 24 & $89.85 \pm 24.7$ & 152 & 66.65 & 82 & 79.6 & 81.9 \\
$2008(151)$ & 1 & 103 & $89.69 \pm 27.9$ & 105 & 65.73 & 70 & 65.2 & 67.5 \\
$2009(153)$ & 1 & 24 & $90.9 \pm 22.8$ & 126 & 61 & 88 & 87.8 & 87.8 \\
$2010(153)$ & - & 39 & $89.4 \pm 25.9$ & 111 & 68.65 & 80.4 & 79.2 & 79.2 \\
$2011(153)$ & 2 & 46 & $95.4 \pm 20.8$ & 66 & 55.2 & 88.3 & 79.1 & 81.2 \\
Promedio & $0.5 \pm 1.09$ & $35.3 \pm 23$ & $96.76 \pm 5.3$ & $106.4 \pm 27.2$ & $59.15 \pm 6.3$ & $77.9 \pm 12.3$ & $74.4 \pm 11.5$ & $76.3 \pm 12$ \\
\hline
\end{tabular}

*\#N.D.=Nidadas Depredadas; \#N. S.=Nidadas saqueadas, T. N.=Tamaño nidada (huevos), $\mathrm{N}^{\circ}=$ Numero de nidadas; P.I=Período de Incubación; \%Ecl.=9 Éxito de Eclosión, \% Emer.=Éxito de Emergencia, \% Recl.=Éxito de Reclutamiento.

tortuga lora más antiguo de toda la costa Pacífica de Costa Rica.

Durante los primeros diez años de ejecución del proyecto, fue observado que el número promedio de eventos de tortuga lora por temporada fue menor a 250 eventos/año. Para los últimos seis años (2006-2011), este valor se ha mantenido por lo general superior o similar a 250 eventos/año por temporada. Ya que el esfuerzo de muestreo (hombre/noche) por temporada se mantiene invariable en un promedio de 153 noches muestreadas por temporada, este aumento en el número total de eventos de anidación puede ser un indicador de recuperación para la población de tortuga lora en Punta Banco. Luego de 15 años continuos de protección, es de esperar este aumento de eventos, ya que se espera que las primeras tortugas fruto de estos esfuerzos de protección visiten la playa, debido a que la tortuga lora alcanza la madurez sexual entre los 12 y 14 años de edad (Plotkin, 2007).

Los esfuerzos oficiales para conservar las tortugas marinas del Pacífico de Costa Rica se iniciaron durante los años 70, concentrándose en las dos playas (Ostional y Nancite) donde la tortuga lora anida de una manera peculiar conocida como "arribada", fenómeno que consiste en un evento de anidación simultáneo y masivo que dura aproximadamente tres días. $\mathrm{La}$ actividad de anidación de la tortuga lora, sin embargo, ocurre en prácticamente cada playa arenosa del Pacífico costarricense, en una forma descrita como anidación "solitaria". Considerando que la mayoría de las playas de anidación solitaria, así como cualquier playa costarricense que no goce de protección oficial, son completamente saqueadas y ningún huevo es dejado para reabastecer las ya diezmadas poblaciones de tortugas marinas (Arauz, 2002), 
es importante que las medidas de conservación sean incrementadas.

El alto porcentaje de saqueo es una preocupación, especialmente considerando información obtenida a partir de estudios comparativos entre el éxito de eclosión en playas de "arribada" y de anidación "solitaria". Los resultados de estos estudios indican porcentajes muy bajos de eclosión en los sitios donde ocurren arribadas, aparentemente debido a la alta concentración de tortugas que al anidar destruyen mecánicamente los huevos que fueron depositados con anterioridad por otras tortugas. La destrucción masiva de huevos fomenta la proliferación de hongos y bacterias, afectando el desarrollo de los huevos y causando tasas de eclosión muy bajas (de 1\% a 8\%) (Cornelius et al., 1992). En contraste, en las playas de anidación solitaria el éxito de eclosión puede ser considerablemente más alto, hasta de un $80 \%$, un hecho que destaca la importancia de la contribución de la anidación solitaria al mantenimiento de la población de tortuga lora del Pacífico Oriental (Castro, 1986) y la importancia de la protección de las playas de desove.

Adicionalmente, aunque se cree que la tortuga lora es la más abundante de las siete especies de tortugas marinas reconocidas en el mundo, la necesidad de esfuerzos de conservación con respecto a esta especie no debe ser desestimada. Así las medidas de conservación para protegerlas son ahora necesarias para asegurar que no lleguen las poblaciones de esta especie a una situación similar a la que enfrenta la tortuga baula en el Pacífico Oriental (sus poblaciones anidadoras del mundo han disminuído en un $95 \%$ y el Océano Pacífico puede ahora contener tan pocas como 1000 hembras adultas (Crowder, 2000).

El deseo de proteger, conservar y restaurar las poblaciones de tortugas marinas y de sus hábitats, ha llevado a la creación de un gran número de programas de protección en todo el mundo (Eastern Pacific Hawksbill Initiative, ICAPO, Organización Sea Turtle Conservancy, Wider Caribbean Sea Turtle Conservation Network, WIDECAST). En numerosos casos, los pasos iniciales más efectivos han sido entrar a las comunidades cercanas a las playas de anidación e informar a sus miembros sobre el estado crítico de las poblaciones de tortugas marinas y disuadirles del consumo de productos provenientes de ellas (huevos, carne, caparazón, etc.). Cada comunidad costera se beneficia directamente del recurso y son ellos finalmente los responsables de la protección de la playa y sus recursos, por lo que es necesario llevarles alternativas a sus prácticas y darles incentivos contra la explotación de los productos de tortuga marina, pues, como se ha establecido ya, las tortugas marinas valen mucho más en una comunidad costera vivas que muertas (Troëng \& Drews, 2004). El involucramiento de los locales (pescadores, miembros de comunidades costeras) se debe traducir en trabajos, responsabilidades, entrenamiento técnico y educación, integrando el desarrollo de la comunidad con la conservación de los recursos naturales en un plan a largo plazo, mediante procesos participativos e inclusivos

El programa de protección desarrollado por la ONG PRETOMA en playa Punta Banco, ha contado desde un principio con la colaboración de la comunidad local, se contratan servicios de miembros de las comunidades como monitores de playa, entrenándoles y asesorándoles para una mejor protección en la playa. Además PRETOMA también trabaja con estudiantes de pasantía (promedio de cinco por temporada) como coordinadores de investigación y con voluntarios nacionales y extranjeros (promedio entre 50 a 100 personas por temporada, además de grupos). En este sentido, es importante fomentar la visita de voluntarios y turistas a Punta Banco, durante las temporadas de anidación de tortugas, ya que este aumento significa la posibilidad de mejorar el patrullaje y el monitoreo de la playa. Además ofrece a miembros de la comunidad la oportunidad de hacer amigos "internacionales" y desarrollar actividades productivas (comidas, bebidas, hoteles, estadías en casas, servicio de taxi, alquiler de caballos). El fortalecimiento del programa de voluntarios incidiría en más beneficios económicos para miembros de la comunidad. 
La principal técnica de manejo usada en Punta Banco en la protección de las nidadas hasta la fecha, ha sido la operación de viveros. Existe preocupación en el uso de viveros especialmente en cuanto a la influencia de la temperatura sobre la determinación del sexo así como en qué grado los neonatos son afectados por la intervención y la manipulación humana en el proceso de "impronta", un comportamiento que permite a los neonatos encontrar su playa natal cuando anidan como adultos.

Los viveros, sin embargo, ofrecen ciertos beneficios indiscutibles, especialmente como instrumentos de educación ambiental, permitiendo a los miembros de la comunidad ver directamente el producto de sus esfuerzos. Niños escolares, miembros de comunidades locales y voluntarios del proyecto cuentan neonatos y participan en su liberación al anochecer. Estas actividades estimulan a los miembros de la comunidad para continuar con estos esfuerzos de protección. Por el momento, se utiliza un enfoque precautorio, procurando minimizar la manipulación de huevos y neonatos. Mortimer (1999) estableció que los porcentajes de éxito de eclosión de nidos puestos en viveros son generalmente menores de esos que son dejados en su estado natural, sin embargo este no es el caso en los viveros de PRETOMA, en los que el éxito de eclosión se ha mantenido en un valor promedio de $77.9 \%$. A pesar del trabajo de concientización llevado a cabo en la comunidad, la costumbre de usar productos de tortuga marina está arraigada en años de tradición cultural, y esperar que esas tradiciones cambien rápidamente es irreal. Además miembros de otras comunidades próximas a Punta Banco usan esta playa para saquear los huevos, ya que conocen de la falta de protección gubernamental, por lo que, la operación de viveros puede ser la única manera de reducir significativamente el saqueo de nidos en proyectos de conservación de tortugas marinas que se desarrollan en comunidades costeras.

El promedio de remigración de L. olivácea en playa Punta Banco, fue de 1.9 años. Se han observado tortugas que vuelven en diferentes años a anidar a la misma playa Punta Banco.
El periodo de remigración más largo que se ha observado es una tortuga marcada en el 2001 y reobservada de nuevo en el 2006, es decir, cinco años. Del total de tortugas reanidantes, se han observado cuatro tortugas que anidaron primero en otro proyecto de PRETOMA en Guanacaste, Pacífico Norte de Costa Rica y a continuación se trasladaron a anidar en Punta Banco en el Pacífico Sur. El porcentaje de reobservación en el proyecto de playa Punta Banco se ha mantenido cada año en un promedio de $20 \%$, lo que significa que el $80 \%$ de las tortugas que se marcan, no vuelven a verse durante esa temporada. El monitoreo realizado por el personal de PRETOMA no abarca todo el área posible de anidación de esta zona, ya que al final del pueblo se extiende más área de playa en una extensa reserva indígena con difícil acceso que no es muestreada. Este puede ser un motivo para que no se vean todas las tortugas cuando salen a anidar. La amenaza más inmediata a la supervivencia de las tortugas y que puede explicar la baja tasa de hembras reanidantes es el alto número de capturas incidentales en las flotas camaroneras. La tortuga lora es la especie de tortuga más comúnmente capturada $(90 \%)$ y la tortuga verde, la menos capturada $(9.6 \%)$. La mortalidad de las tortugas capturadas por estas redes se estima en alrededor del $40 \%$ para la tortuga lora y en un $50 \%$ para la tortuga verde (Arauz, Vargas, Naranjo \& Gamboa, 1998). Es común que las tortugas mueran o se lastimen durante este proceso, especialmente en profundidades someras y medias (Arauz \& Ballestero, 2003). En Costa Rica, la flota camaronera de arrastre captura anualmente más de 15000 tortugas marinas (Arauz et al., 1998)

El área marina frente a la playa de anidación Punta Banco corresponde al Golfo Dulce, una de las zonas más importantes en biodiversidad marina de la costa pacífica de Costa Rica (Morales-Ramírez, 2011) y de gran importancia para su protección, tanto la zona marina como la playa. Actualmente se está trabajando con los pescadores de la zona de Punta Banco y alrededores para establecer un Área Marina Protegida y poder brindar protección 
no solo a las tortugas marinas sino a todos los recursos marinos de la zona. Para luchar contra las amenazas que sufre la tortuga lora en esta área (extracción ilegal de huevos, pesca de arrastre, degradación del litoral costero, granjas atuneras etc.), se están implementando estrategias de gestión integrada promovidas por la Comisión ACOSA, así como colaboración entre las organizaciones medioambientales y las comunidades locales.

En la temporada actual 2013-2014, el comité de desarrollo de playa Punta Banco, en apoyo con un programa de ayuda del IMAS ha comenzando un proyecto propio de monitoreo y protección de esta playa, para evitar así que el saqueo aumente en los próximos años y poder mantener la población de tortugas marinas de playa Punta Banco.

\section{AGRADECIMIENTOS}

Agradecemos a todos los coordinadores, asistentes de campo y voluntarios nacionales e internacionales que participaron en el proyecto, en especial a Pau Sunyer por todo su apoyo y trabajo, a Alexander Gaos, Ingrid Yañez, Jose Cortés, Maikol Vargas, Charlotte Adams y todo el personal de PRETOMA. Agradecemos también el apoyo del pueblo de Punto Banco. Se agradece al Proyecto Consolidación de Áreas Marinas Protegidas del Sistema Nacional de Áreas de Conservación, Programa de Naciones Unidas para el Desarrollo y el Global Environment Facility (GEF) por su colaboración en la publicación.

\section{RESUMEN}

Se realizó un monitoreo de la actividad de anidación de tortugas marinas en Punta Banco, Pacífico Sur de Costa Rica, de forma ininterrumpida por diez y seis años, acompañado por actividades de conservación como la relocalización de los nidos en viveros. Se realizaron análisis de éxito de eclosión para nidos "in situ" y en vivero. Evaluamos por este medio la biología reproductiva de la tortuga lora (Lepidochelys olivacea), por mucho la tortuga más común que anida en Punta Banco $(98 \%$ de las tortugas observadas). Entre 1996 y 2011, se llevó a cabo el monitoreo diario de las actividades de anidación de julio a diciembre. Marcamos un total de 1239 tortugas L. olivacea y registramos 4130 eventos de anidación. El total de anidaciones para L. olivacea varió de 239 en el año 1996 a 402 en 1999, mientras que el número de hembras avistadas varió de 18 en 1996 a 146 en 1999. Las hembras presentaron un largo curvo del caparazón promedio de $66.8 \mathrm{~cm}$ y ancho curvo de caparazón promedio de $70.7 \mathrm{~cm}$. La media para el tamaño de las nidadas fue de 96.7 huevos, con una frecuencia de puesta observada de 1.07 nidos/hembra y un intervalo de puesta observado de 19.95 días. El éxito de eclosión de nidos "in situ" fue de $61.38 \%$, mientras que el éxito de nidos relocalizados en viveros fue de $77.9 \%$. La población de L. olivacea en Punta Banca muestra una tendencia positiva.

Palabras clave: Tortuga marina, playa de anidación, Lepidochelys olivacea, costa pacífica de Costa Rica.

\section{REFERENCIAS}

Alvarado, J., \& Murphy, T. M. (1999). Nesting Periodicity and Internesting Behavior. In K. L. Eckert, K. A. Bjorndal, F. A. Abreu-Grobois \& M. Donnelly (Eds.), Research and Management Techniques for the Conservation of Sea Turtles (pp. 115-118). Washington D.C., USA: Marine Turtle Specialist Group, IUCN/ SSC.

Arauz, R., Vargas, R., Naranjo, I., \& Gamboa, C. (1998). Analysis of the incidental capture and mortality of sea turtles in the shrimp fleet of Pacific Costa Rica. In S. P. Epperly \& J. Braun (Eds.), Proceedings of the Seventeenth Annual Sea Turtle Symposium (pp. 294). USA: NOAA Technical Memorandum NMFS-SEFSC-415.

Arauz, R. (2002). Sea turtle nesting activity and conservation of leatherback sea turtles (Dermochelys coriacea) in Playa El Mogote, Río Escalante Chacocente Wildlife Refuge, Nicaragua. Managua, Nicaragua: Sea Turtle Restoration Project/Central American Office.

Arauz, R., \& Ballestero, J. (2003). Estudio sobre el diseño del Dispositivo Excluidor de Tortugas (DET) con observadores a bordo del 5\% de la flota camaronera, que permita incrementar la eficiencia de la operación en al menos un $85 \%$, definir áreas de uso, y obtener información biométrica para determinar la apertura del DET (Informe Final). Costa Rica: Cámara Puntarenense de Pescadores, PRETOMA.

Bolten, A. (1999). Techniques for Measuring Sea Turtles. In K. L. Eckert, K. A. Bjorndal, F. A. Abreu-Grobois \& M. Donnelly (Eds.), Research and Management Techniques for the Conservation of Sea Turtles (pp 104-114). Washington D.C., USA: Marine Turtle Specialist Group, IUCN/SSC. 
Bjorndal, K. A., Wetherall, J. A., Bolten, A. B., \& Mortimer, J. A. (1999). Twenty-six years of green turtle nesting at Tortuguero, Costa Rica: an encouraging trend. Conservation Biology, 13, 126-134.

Broderick, A. C., Franenstein, R., Glen, F., \& Hays, G. C. (2006). Are green turtles globally endangered? Global Ecology and Biogeography, 15, 21-26.

Castro, J. C. (1986). Contribución de las tortugas loras solitarias (Lepidochelys olivacea Eschscholtz) en el mantenimiento de las poblaciones de esta especie (Tesis de Licenciatura). Universidad de Costa Rica, Costa Rica.

CIT. (Setiembre, 2008). Manual Sobre Técnicas de Manejo y Conservación de las Tortugas Marinas en Playas de Anidación de Centroamérica. Taller de capacitación sobre técnicas de manejo y conservación de tortugas marinas en playas de anidación en la región centroamericana. Tortuguero, Costa Rica: CIT.

Cornelius, S. E., Alvarado, M., Castro, J. C., Mata, M., \& Robinson, D. C. (1992). Management of olive ridley sea turtles (Lepidochelys olivacea) nesting at Playas Nancite and Ostional, Costa Rica. In J. G. Robinson \& K. H. Redford (Eds.), Neotropical Wildlife Use and Conservation (pp. 111-135). Chicago IL: The University of Chicago Press.

Crowder, L. (2000). Leatherback's survival will depend on an international effort. Nature, 405.

Eckert, K. L., Bjorndal, K. A., Abreu-Grobois, F. A., \& Donnelly, M. (2000). Técnicas de investigación y manejo para la conservación de las tortugas marinas. Washington D.C., USA: UICN/CSE Grupo Especialista en Tortugas Marinas.

Ferrer, Y., Díaz, R., \& Díaz, F. R. (2007). Características de la anidación de la tortuga verde, Chelonia mydas (Testudinata, Cheloniidae), en la playa Caleta de los Piojos, Cuba, a partir de marcaciones externas. $A B C$, 30, 211-218

Godfrey, M. H., \& Mrosovsky, N. (1997). Estimating the time between hatching of sea turtles and their emergence from the nest. Chelonian Conservation and Biology, 2, 581-585.

Limpus, C. J. (1995). Global overview of the status of marine turtles: a 1995 viewpoint. In K. A. Bjorndal (Ed.), Biology and Conservation of Sea Turtles, Revised edition (pp. 605-609). Washington D.C.: Smithsonian Institution Press.

Miller, J. D., (1997). Reproduction in sea turtles. In P. L. Lutz \& J. A. Musik (Eds.), The 1 Biology of the Sea Turtles (pp. 51-80). Boca Ratón, USA: CRC Press.

Miller, J. D. (2000). Determining Clutch Size and Hatching Success. In K. L. Eckert, K. A. Bjorndal, F. A.
Abreu-Grobois \& M. Donnelly (Eds.), Research and Management Techniques for the Conservation of Sea Turtles (pp. 51-80). Washington DC: Marine Turtle Specialist Group, IUCN/SSC.

Morales-Ramírez, A. (2011). La diversidad marina del Golfo Dulce, Pacífico Sur de Costa Rica: amenazas a su conservación. Biocenosis, 24, 1-2.

Mortimer, J. (1999). Reducing threats to eggs and hatchlings: Hatcheries, In K. L. Eckert, K. A. Bjorndal, F. A. Abreu-Grobois \& M. Donnelly (Eds.), Research and Management Techniques for the Conservation of Sea (pp. 175-181). Washington DC: Marine Turtle Specialist Group, IUCN/SSC.

Piedra, R., Vélez, E., Dutton, P., Possardt, E., \& Padilla, C. (2007). Nesting of the Leatherback turtle (Dermochelys coriacea) from 1999-2000 through 2003-2004 at Playa Langosta, Parque Nacional Marino Las Baulas de Guanacaste, Costa Rica. Chelonian Conservation and Biology, 6, 111-116.

Plotkin, P. (2007). Biology and conservation of Ridley Sea Turtles. Baltimore, MD: Johns Hopkins University Press.

Rees, A., Saad, A., \& Jony, M. (2005). Clutch size and hatching success of green turtle nests in Syria during 2004. In A. Demetropoulos \& O. Turkozan (Eds.), Proceedings of the Second Mediterranean Conference on Marine Turtles (pp. 158-161). Kemer: Turkey.

Russo, M., \& Girondot, M. (2009). How long to monitor marine turtle populations to conclude 15 for a trend? (Report to SWOT). Paris, France: Laboratoire Ecologie, Systématique et Evolution, Centre National de la Recherche Scientifique et Université Paris.

Sarti-Martínez, L. (2002). Acuerdos sobre la estandarización de métodos, términos y definiciones usados en tortugas marinas. Mimeografiado, 9.

Sarti-Martínez, L., Barragán, A. R., García-Muñoz, D., García, N., Huerta, P., \& Vargas, F. (2007). Conservation and Biology of the Leatherback Turtle in the Mexican Pacific. Chelonian Conservation and Biology, 6, 70-78.

Schroeder, B., \& Murphy, S. (1999). Population surveys on nesting beaches. In K. L. Eckert, K. A. Bjorndal, F. A. Abreu-Grobois \& M. Donnelly (Eds.), Research and Management Techniques for the Conservation of Sea Turtles (pp. 45-55). Washington DC: Marine Turtle Specialist Group, IUCN/SSC.

Spotila, J. R. (2004). Sea turtles: A complete guide to their biology, behavior, and conservation. Baltimore, MD: Johns Hopkins University Press. 
Troëng, S., \& Drews, C. (2004). Money Talks: Economic Aspects of Marine Turtle Use and Conservation. Retrieved from www.panda.org

Unión Internacional para la Conservación de la Naturaleza (UICN). (2013). The IUCN Red List of Threatened Species. Version 2010.4. Retrieved form www.iucnredlist.org

Vera, V., \& Buitrago, J. (2012). Actividad Reproductiva de Chelonia mydas (Testudines: Cheloniidae) en Isla de
Aves, Venezuela (2001-2008). Revista de Biología Tropical, 60, 2.

Viejobueno, M. S., Adams, C., \& Arauz, R. (2013). Conservación e investigación de tortugas marinas en el Pacifico de Costa Rica. (Refugio Nacional de Vida Silvestre Caletas-Arío, San Miguel, Corozalito) (Reporte técnico). Costa Rica: Ministerio de Ambiente, Energía y Telecomunicaciones (MINAET). 